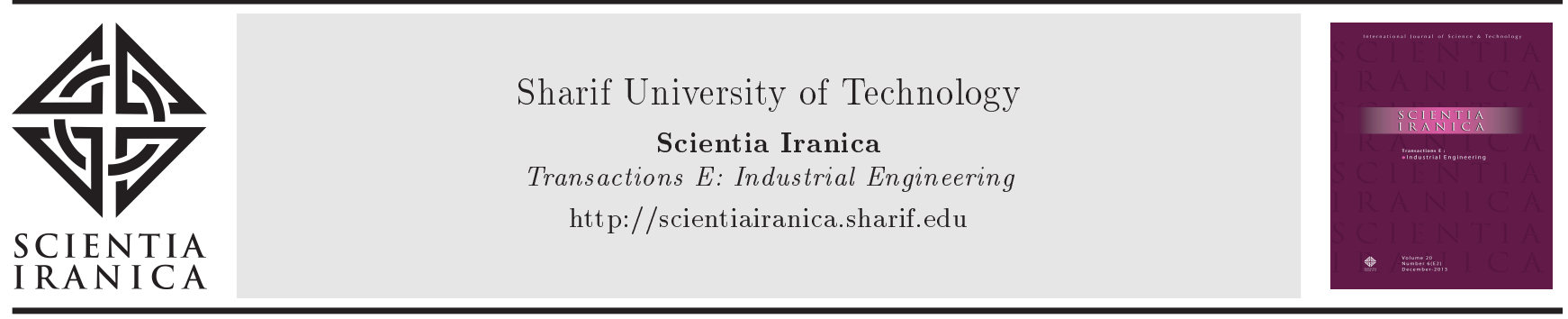

Research Note

\title{
Reliability analysis of three-component mixture of distributions
}

\author{
M. Aslam ${ }^{\mathrm{a}}$, M. Tahir ${ }^{\mathrm{b} *}$, and Z. Hussain ${ }^{\mathrm{c}}$ \\ a. Department of Mathematics and Statistics, Riphah International University, Islamabad 44000, Pakistan. \\ b. Department of Statistics, Government College University, Faisalabad 38000, Pakistan. \\ c. Department of Statistics, Quaid-i-Azam University, Islamabad 44000, Pakistan.
}

Received 3 November 2015; received in revised form 13 October 2016; accepted 28 January 2017

\section{KEYWORDS}

Three-component mixture distributions;

Reliability analysis;

Failure rate function;

Mean residual life

function;

Mean waiting time

function.

\begin{abstract}
This article focuses on studying three-component mixture of Exponential, Rayleigh, Pareto, and Burr Type-XII distributions in relation to reliability analysis. The main purpose of this study is to derive algebraic expressions for different functions of survival time. For these three-component mixtures of distributions, the cumulative distribution function, hazard rate function, cumulative hazard rate function, reversed hazard rate function, mean residual life function, and mean waiting time function are discussed. To study the behaviors of different reliability functions, numerical results are presented for fixed values of parameters.
\end{abstract}

(C) 2018 Sharif University of Technology. All rights reserved.

\section{Introduction}

Reliability analysis is a tool kit of statistical procedures for analyzing time-to-failure data. Usually, reliability analysis is carried out using the classical or Bayesian statistical analysis of parametric reliability models. Reliability analysis datasets are represented with a single univariate or multivariate statistical distributions such as Exponential, Rayleigh, inverse Weibull, Pareto and Burr distributions [1-6]. Recently, some other distributions have been used to model the survival data. The use of mixture distributions has obtained popularity for modeling the heterogeneous data. Mixture of distributions is useful because it is applied to represent heterogeneous dataset where there is evidence of multimodality. Chen et al. [7] used a two-component mixture model for the analysis

*. Corresponding author. Tel.: +92 3015237403 E-mail address: tahirqaustat@yahoo.com (M. Tahir).

doi: $10.24200 /$ sci.2017.4441 of cancer survival data generalizing an earlier idea in the study of Berkson and Gage [8]. In Qian's study [9], a similar model of a mixture of a Weibull component and a surviving fraction in the context of a lung cancer trial are considered. Angelis et al. [10] proposed an application of a mixture model to relative survival rates of colon cancer patients from the Finnish population-based cancer registry including major survival determinants as explicative covariates. Marin et al. [11] illustrated how Bayesian methods can be used to fit a mixture of Weibull models with an unknown number of components to heterogeneous, possibly right-censored survival data using a birth death MCMC algorithm. Abu-Taleb et al. [12] presented the Bayesian estimation of lifetime parameters of Exponential distributions when survival and censoring times are both exponentially distributed. Erişoğlu et al. [13] studied the mixture model of two different distributions to analyze the heterogeneous survival data. Krishna and Malik [14] presented the reliability estimation in Maxwell distribution using progressively type-II censored data. Ali [15] discussed 
the reliability properties of two-component mixture of inverse Rayleigh distributions. Also, a valuable account on the reliability properties and analysis of mixture distributions is given in [16-26], and many others alike.

Similar to two-component mixture modeling, some authors have discussed situations where data are assumed to come from a three-component mixture of distributions. For example, in order to know the proportion of failure due to a certain cause and improve the manufacturing process, Acheson and McElwee [27] divided electronic tube failures into three types of defects: gaseous defects, mechanical defects, and normal deterioration of the cathode. Further, as mentioned by Tahir et al. [28], Davis [29] reported a mixture data on lifetimes of many components used in aircraft sets. To illustrate the proposed methodology for a three-component mixture distribution, Tahir et al. [28] (2016) used the time-to-failure data on three components: transmitter tube, combination of transformers, and combination of relays. Thus, the existence of three-component mixtures of distributions is evident.

There are two approaches to statistical modeling and analyses: a model is assumed to exist in nature to generate data, and parameters of the models are estimated on the basis of these data; data are observed first and a suitable model is fitted to them. The first approach seems more logical. Following the first approach, it is assumed in this study that there exist models in nature, capable of yielding data as coming from a three-component mixture of distributions. We, specifically, assumed the existence of three-component mixture of Exponential, Rayleigh, Pareto, and Burr Type-XII distributions. For these mixtures of distributions, different reliability functions are studied both algebraically and numerically which is the main purpose of this article. This study may be useful for the practitioners dealing with the data coming from a three-component mixture of Exponential, Rayleigh, Pareto, and Burr Type-XII distributions.

A random variable, $Y$, is said to follow a finite mixture distribution with $q$ components if the density function of $Y$ can be written in the form:

$$
f(y)=\sum_{m=1}^{q} p_{m} f_{m}(y),
$$

where $p_{m}(m=1,2, \ldots, q)$ is the $m$ th mixing proportion, such that:

$$
p_{q}=1-\sum_{m=1}^{q-1} p_{m}
$$

and $f_{m}(y)$ is the $m$ th component density function. A finite three-component mixture of distributions with mixing proportions $p_{1}$ and $p_{2}$ has the probability density function (pdf) as follows:

$$
\begin{aligned}
f(y ; \Psi)= & p_{1} f_{1}\left(y ; \Psi_{1}\right)+p_{2} f_{2}\left(y ; \Psi_{2}\right) \\
& +\left(1-p_{1}-p_{2}\right) f_{3}\left(y ; \Psi_{3}\right) \\
& p_{1}, p_{2} \geq 0, p_{1}+p_{2} \leq 1
\end{aligned}
$$

where, $\Psi=\left(\lambda_{1}, \lambda_{2}, \lambda_{3}, p_{1}, p_{2}\right), \Psi_{m}=\lambda_{m}, m=1,2,3$, and $f_{m}\left(y ; \Psi_{m}\right)$ is the pdf of the $m$ th component density. The cumulative distribution function (cdf) for a finite three-component mixture distribution can be written as follows:

$$
\begin{aligned}
F(y ; \Psi) & =p_{1} F_{1}\left(y ; \Psi_{1}\right) \\
& +p_{2} F_{2}\left(y ; \Psi_{2}\right)+\left(1-p_{1}-p_{2}\right) F_{3}\left(y ; \Psi_{3}\right),
\end{aligned}
$$

where $F_{m}\left(y ; \Psi_{m}\right)$ is the cdf of the $m$ th component density.

\section{Different functions of survival time}

In reliability theory, classification of lifetime models is defined in terms of different functions of survival time such as reliability (survival), failure rate (hazard rate), cumulative hazard rate, reversed hazard rate, mean residual life, and mean waiting time functions. We now define these functions for a three-component mixture of distributions.

Let $y$ be the survival time. The reliability function for a three-component mixture of distributions is defined as:

$$
\begin{aligned}
R(y ; \Psi)= & 1-\int_{0}^{y} f(u ; \Psi) d u=1-F(y ; \Psi), \\
R(y ; \Psi)= & p_{1} R_{1}\left(y ; \Psi_{1}\right)+p_{2} R_{2}\left(y ; \Psi_{2}\right) \\
& +\left(1-p_{1}-p_{2}\right) R_{3}\left(y ; \Psi_{3}\right),
\end{aligned}
$$

where $R_{m}\left(y ; \Psi_{m}\right)$ is the reliability function of the $m$ th component.

The hazard rate function for a three-component mixture of distributions is defined as ratio of lifetime model to reliability function:

$$
\begin{aligned}
& h(y ; \Psi)=\frac{f(y ; \Psi)}{R(y ; \Psi)} \\
& =\frac{p_{1} f_{1}\left(y ; \Psi_{1}\right)+p_{2} f_{2}\left(y ; \Psi_{2}\right)+\left(1-p_{1}-p_{2}\right) f_{3}\left(y ; \Psi_{3}\right)}{p_{1} R_{1}\left(y ; \Psi_{1}\right)+p_{2} R_{2}\left(y ; \Psi_{2}\right)+\left(1-p_{1}-p_{2}\right) R_{3}\left(y ; \Psi_{3}\right)} .
\end{aligned}
$$

The cumulative hazard rate function, $H(y ; \Psi)$, and reversed hazard rate function, $r(y ; \Psi)$, for a threecomponent mixture of distributions are given by: 
$H(y ; \Psi)=\int_{0}^{y} h(u ; \Psi) d u=-\ln \{1-F(y ; \Psi)\}=-\ln R(y ; \Psi)$,

and:

$r(y ; \Psi)=\frac{f(y ; \Psi)}{F(y ; \Psi)}$

$=\frac{p_{1} f_{1}\left(y ; \Psi_{1}\right)+p_{2} f_{2}\left(y ; \Psi_{2}\right)+\left(1-p_{1}-p_{2}\right) f_{3}\left(y ; \Psi_{3}\right)}{p_{1} F_{1}\left(y ; \Psi_{1}\right)+p_{2} F_{2}\left(y ; \Psi_{2}\right)+\left(1-p_{1}-p_{2}\right) F_{3}\left(y ; \Psi_{3}\right)}$.

In case $\frac{H(y ; \Psi)}{y}$ is decreasing (increasing), one obtains the decreasing failure rate average (increasing failure rate average) family since the average failure rate in that case will be decreasing (increasing).

The Mean Residual Life (MRL) function at a given time $y$ measures the expected remaining life time of an individual of age $y$. It is denoted by $m(y ; \Psi)$. The MRL function or life expectancy function for a three-component mixture of distributions is defined as follows:

$$
\begin{aligned}
& m(y ; \Psi)=\frac{1}{R(y ; \Psi)} \int_{y}^{\infty} u f(u ; \Psi) d u-y, \\
& m(y ; \Psi)=\frac{1}{R(y ; \Psi)}\left\{E(Y)-\int_{0}^{y} u f(u ; \Psi) d u\right\}-y .
\end{aligned}
$$

Increasing (decreasing) mean residual life function $m(y ; \Psi)$ produces the family of increasing mean residual life (decreasing mean residual life) distributions.

Another important function in reliability analysis is of Mean Waiting Time (MWT). The MWT function is known as expected inactivity time function. For a three-component mixture of distributions, the MWT function of an item failed in an interval $[0, y]$ is defined as follows:

$$
\bar{\mu}(y ; \Psi)=y-\left\{\frac{1}{F(y ; \Psi)} \int_{0}^{y} u f(u ; \Psi) d u\right\} .
$$

\section{Reliability properties of a three-component mixture of exponential distributions}

Using Eqs. (1) and (2), a finite three-Component Mixture of Exponential Distributions (3-CMED) with mixing proportions $p_{1}$ and $p_{2}$ has the pdf and cdf as follows:

$$
\begin{aligned}
& f(y ; \Psi)=p_{1} \lambda_{1} \exp \left(-\lambda_{1} y\right)+p_{2} \lambda_{2} \exp \left(-\lambda_{2} y\right) \\
& +\left(1-p_{1}-p_{2}\right) \lambda_{3} \exp \left(-\lambda_{3} y\right), \\
& 0<y<\infty, \quad \lambda_{m}>0, \quad m=1,2,3, \\
& F(y ; \Psi)=1-p_{1} \exp \left(-\lambda_{1} y\right)-p_{2} \exp \left(-\lambda_{2} y\right) \\
& -\left(1-p_{1}-p_{2}\right) \exp \left(-\lambda_{3} y\right) .
\end{aligned}
$$

\subsection{Reliability and failure rate functions for a 3-CMED}

The reliability function or survival function for a 3CMED is written as:

$$
\begin{aligned}
& R(y ; \Psi)= p_{1} \exp \left(-\lambda_{1} y\right)+p_{2} \exp \left(-\lambda_{2} y\right) \\
&+\left(1-p_{1}-p_{2}\right) \exp \left(-\lambda_{3} y\right) \\
& 0<y<\infty, \quad \lambda_{m}>0, \quad m=1,2,3
\end{aligned}
$$

The failure rate function or hazard rate function for a 3-CMED is defined as shown in Box I.

The behavior of hazard rate function for the 3-CMED for some fixed values of component and proportion parameters is depicted in Figures 1-3 in which the effect of parameters $\lambda_{1}, \lambda_{2}, \lambda_{3}, p_{1}$ and $p_{2}$ on hazard rate for the 3 -CMED can be observed. These graphs also explain the flexibility of the hazard rate for a 3-CMED.

Using the expression in Eq. (12), the hazard rate of a 3 -CMED is evaluated for the parametric values fixed in Figures 1-3. The numerical results, so obtained, are presented in Table 1.

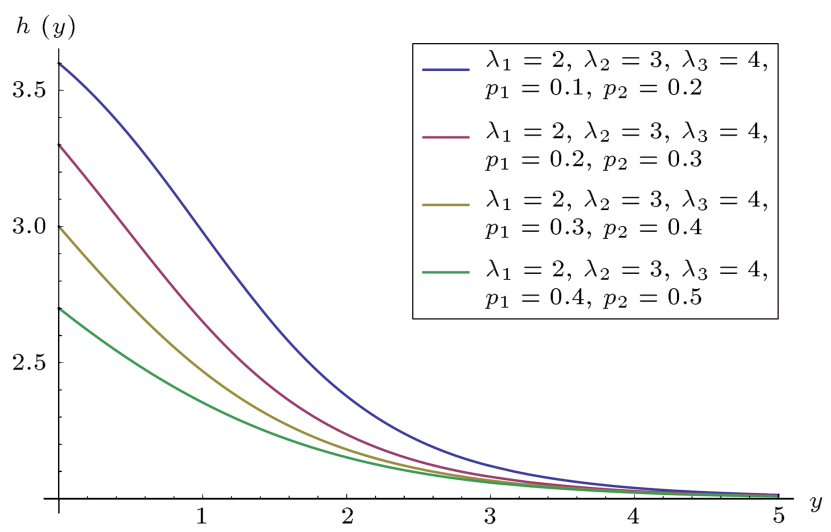

Figure 1. Graphs of hazard rate function for a 3-CMED for parameters $\left\{\left(\lambda_{1}, \lambda_{2}, \lambda_{3}, p_{1}, p_{2}\right)=(2,3,4,0.1,0.2),(2\right.$, $3,4,0.2,0.3),(2,3,4,0.3,0.4),(2,3,4,0.4,0.5)\}$.

$$
h(y ; \Psi)=\frac{p_{1} \lambda_{1} \exp \left(-\lambda_{1} y\right)+p_{2} \lambda_{2} \exp \left(-\lambda_{2} y\right)+\left(1-p_{1}-p_{2}\right) \lambda_{3} \exp \left(-\lambda_{3} y\right)}{p_{1} \exp \left(-\lambda_{1} y\right)+p_{2} \exp \left(-\lambda_{2} y\right)+\left(1-p_{1}-p_{2}\right) \exp \left(-\lambda_{3} y\right)} .
$$


$H(y ; \Psi)=-\ln \left\{p_{1} \exp \left(-\lambda_{1} y\right)+p_{2} \exp \left(-\lambda_{2} y\right)+\left(1-p_{1}-p_{2}\right) \exp \left(-\lambda_{3} y\right)\right\}$

and

$r(y ; \Psi)=\frac{p_{1} \lambda_{1} \exp \left(-\lambda_{1} y\right)+p_{2} \lambda_{2} \exp \left(-\lambda_{2} y\right)+\left(1-p_{1}-p_{2}\right) \lambda_{3} \exp \left(-\lambda_{3} y\right)}{1-p_{1} \exp \left(-\lambda_{1} y\right)-p_{2} \exp \left(-\lambda_{2} y\right)-\left(1-p_{1}-p_{2}\right) \exp \left(-\lambda_{3} y\right)}$

Box II

$$
m(y ; \Psi)=\frac{p_{1} \frac{1}{\lambda_{1}} \exp \left(-\lambda_{1} y\right)+p_{2} \frac{1}{\lambda_{2}} \exp \left(-\lambda_{2} y\right)+\left(1-p_{1}-p_{2}\right) \frac{1}{\lambda_{3}} \exp \left(-\lambda_{3} y\right)}{p_{1} \exp \left(-\lambda_{1} y\right)+p_{2} \exp \left(-\lambda_{2} y\right)+\left(1-p_{1}-p_{2}\right) \exp \left(-\lambda_{3} y\right)}
$$

Box III

From Figures 1-3 and the entries in Table 1, it is obvious that, in general, failure rate for a 3 CMED follows a decreasing trend over time. When $\lambda_{1}<\lambda_{2}<\lambda_{3}$, failure rate decreases as proportion parameters increase (see row 1 of Table 1). On the

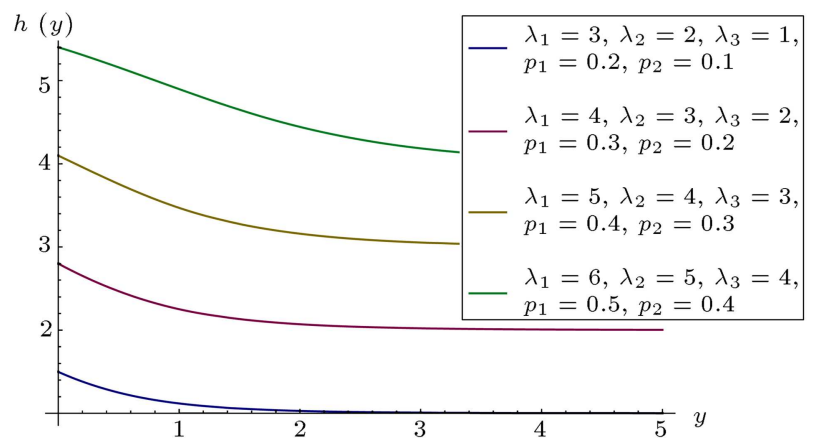

Figure 2. Graphs of hazard rate function for a 3-CMED for parameters $\left\{\left(\lambda_{1}, \lambda_{2}, \lambda_{3}, p_{1}, p_{2}\right)=(3,2,1,0.2,0.1),(4\right.$, $3,2,0.3,0.2),(5,4,3,0.4,0.3),(6,5,4,0.5,0.4)\}$.

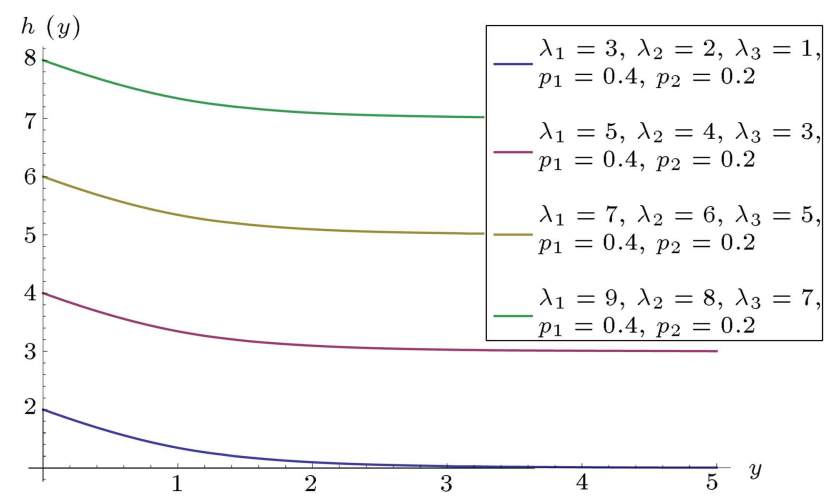

Figure 3. Graphs of hazard rate function for a 3-CMED for parameters $\left\{\left(\lambda_{1}, \lambda_{2}, \lambda_{3}, p_{1}, p_{2}\right)=(3,2,1,0.4,0.2),(5\right.$, $4,3,0.4,0.2),(7,6,5,0.4,0.2),(9,8,7,0.4,0.2)\}$. other hand, when $\lambda_{1}<\lambda_{2}<\lambda_{3}$, failure rate increases as component parameters increase (see rows 2 and 3 of Table 1). Also, there are higher chances of failure when both the component and proportion parameters are relatively larger (see row 2 of Table 1 ).

\subsection{Cumulative hazard rate and reversed hazard rate functions for a 3-CMED}

The cumulative hazard rate function, $H(y ; \Psi)$, and reversed hazard rate function, $r(y ; \Psi)$, for a 3 -CMED using Eqs. (5) and (6) are obtained by Eqs. (13) and (14), as shown in Box II.

\subsection{Mean residual life and mean waiting time functions for a 3-CMED}

The MRL function or life expectancy function for a 3CMED is obtained by Eq. (15) as shown in Box III. Using the above expression Eq. (15), the MRL of a 3CMED is evaluated for the parametric values fixed in

Table 1. Hazard rate of a 3-CMED.

\begin{tabular}{cccc}
\hline $\boldsymbol{\lambda}_{\mathbf{1}}, \boldsymbol{\lambda}_{\mathbf{2}}, \boldsymbol{\lambda}_{\mathbf{3}}, \boldsymbol{p}_{\mathbf{1}}, \boldsymbol{p}_{\mathbf{2}}$ & $\boldsymbol{Y}=\mathbf{1}$ & $\boldsymbol{Y}=\mathbf{5}$ & $\boldsymbol{Y}=\mathbf{1 0}$ \\
\hline $2,3,4,0.1,0.2$ & 2.980380 & 2.013920 & 2.000090 \\
$2,3,4,0.2,0.3$ & 2.649940 & 2.010230 & 2.000070 \\
$2,3,4,0.3,0.4$ & 2.468170 & 2.008990 & 2.000060 \\
$2,3,4,0.4,0.5$ & 2.353170 & 2.008370 & 2.000050 \\
& & & \\
$3,2,1,0.2,0.1$ & 1.119030 & 1.000990 & 1.000010 \\
$4,3,2,0.3,0.2$ & 2.252010 & 2.002740 & 2.000020 \\
$5,4,3,0.4,0.3$ & 3.470680 & 3.006810 & 3.000050 \\
$6,5,4,0.5,0.4$ & 4.897300 & 4.026680 & 4.000180 \\
& & & \\
$3,2,1,0.4,0.2$ & 1.344590 & 1.003450 & 1.000020 \\
$5,4,3,0.4,0.2$ & 3.344590 & 3.003450 & 3.000020 \\
$7,6,5,0.4,0.2$ & 5.344590 & 5.003450 & 5.000020 \\
$9,8,7,0.4,0.2$ & 7.344590 & 7.003450 & 7.000020 \\
\hline
\end{tabular}




$$
\bar{\mu}(y ; \Psi)=\frac{y-\frac{p_{1}}{\lambda_{1}}\left\{\exp \left(-\lambda_{1} y\right)-1\right\}-\frac{p_{2}}{\lambda_{2}}\left\{\exp \left(-\lambda_{2} y\right)-1\right\}-\frac{\left(1-p_{1}-p_{2}\right)}{\lambda_{3}}\left\{\exp \left(-\lambda_{3} y\right)-1\right\}}{1-p_{1} \exp \left(-\lambda_{1} y\right)-p_{2} \exp \left(-\lambda_{2} y\right)-\left(1-p_{1}-p_{2}\right) \exp \left(-\lambda_{3} y\right)} .
$$

\section{Box IV}

Figures 1-3. The numerical results, so obtained, are showcased in Table 2.

From the entries in Table 2, it is seen that, in general, MRL for a 3-CMED follows an increasing trend over time. When $\lambda_{1}<\lambda_{2}<\lambda_{3}$, MRL increases as proportion parameters increase (see row 1 of Table 2 ). On the other hand, when $\lambda_{1}<\lambda_{2}<\lambda_{3}$, MRL decreases as component parameters increase (see rows 2 and 3 of Table 2). Using Eq. (8), the MWT function for a 3-CMED is obtained by Eq. (16) as shown in Box IV.

Using Eq. (16), the MWT of a 3-CMED is evaluated for the parametric values fixed in Figures 13. The numerical results, so obtained, are given in Table 3 .

From the entries in Table 3, it is observed that, in general, MWT for a 3-CMED follows an increasing trend over time. When $\lambda_{1}<\lambda_{2}<\lambda_{3}$, MWT increases as proportion parameters increase (see row 1 of Table 2). On the other hand, when $\lambda_{1}<\lambda_{2}<\lambda_{3}$, MWT decreases as component parameters increase (see rows 2 and 3 of Table 2).

\section{Reliability properties of a three-component mixture of Rayleigh distributions}

Using Eqs. (1) and (2), a finite three-component mixture of Rayleigh distributions (3-CMRD) with mixing

Table 2. MRL of a 3-CMED.

\begin{tabular}{cccc}
\hline $\boldsymbol{\lambda}_{\mathbf{1}}, \boldsymbol{\lambda}_{\mathbf{2}}, \boldsymbol{\lambda}_{\mathbf{3}}, \boldsymbol{p}_{\mathbf{1}}, \boldsymbol{p}_{\mathbf{2}}$ & $\boldsymbol{Y}=\mathbf{1}$ & $\boldsymbol{Y}=\mathbf{5}$ & $\boldsymbol{Y}=\mathbf{1 0}$ \\
\hline $2,3,4,0.1,0.2$ & 0.366027 & 0.497706 & 0.499985 \\
$2,3,4,0.2,0.3$ & 0.406593 & 0.498304 & 0.499989 \\
$2,3,4,0.3,0.4$ & 0.428908 & 0.498505 & 0.499990 \\
$2,3,4,0.4,0.5$ & 0.443027 & 0.498605 & 0.499991 \\
& & & \\
$3,2,1,0.2,0.1$ & 0.952296 & 0.999511 & 0.999997 \\
$4,3,2,0.3,0.2$ & 0.463508 & 0.499545 & 0.499997 \\
$5,4,3,0.4,0.3$ & 0.297994 & 0.332768 & 0.333330 \\
$6,5,4,0.5,0.4$ & 0.208717 & 0.248670 & 0.249991 \\
& & & \\
$3,2,1,0.4,0.2$ & 0.861899 & 0.998291 & 0.999989 \\
$5,4,3,0.4,0.2$ & 0.308037 & 0.333048 & 0.333331 \\
$7,6,5,0.4,0.2$ & 0.189491 & 0.199885 & 0.199999 \\
$9,8,7,0.4,0.2$ & 0.137111 & 0.142796 & 0.142857 \\
\hline
\end{tabular}

proportions, $p_{1}$ and $p_{2}$, has the pdf and cdf as:

$$
\begin{aligned}
& f(y ; \Psi)=p_{1} \frac{y}{\lambda_{1}^{2}} \exp \left(-\frac{y^{2}}{2 \lambda_{1}^{2}}\right)+p_{2} \frac{y}{\lambda_{2}^{2}} \exp \left(-\frac{y^{2}}{2 \lambda_{2}^{2}}\right) \\
& +\left(1-p_{1}-p_{2}\right) \frac{y}{\lambda_{3}^{2}} \exp \left(-\frac{y^{2}}{2 \lambda_{3}^{2}}\right) \text {, } \\
& 0<y<\infty, \quad \lambda_{m}>0 . \quad m=1,2,3, \\
& F(y ; \Psi)=1-p_{1} \exp \left(-\frac{y^{2}}{2 \lambda_{1}^{2}}\right)-p_{2} \exp \left(-\frac{y^{2}}{2 \lambda_{2}^{2}}\right) \\
& -\left(1-p_{1}-p_{2}\right) \exp \left(-\frac{y^{2}}{2 \lambda_{3}^{2}}\right) \text {. }
\end{aligned}
$$

\subsection{Reliability and failure rate functions for a 3-CMRD}

The reliability function or survival function for a 3CMRD is written as follows:

$$
\begin{gathered}
R(y ; \Psi)=p_{1} \exp \left(-\frac{y^{2}}{2 \lambda_{1}^{2}}\right)+p_{2} \exp \left(-\frac{y^{2}}{2 \lambda_{2}^{2}}\right) \\
+\left(1-p_{1}-p_{2}\right) \exp \left(-\frac{y^{2}}{2 \lambda_{3}^{2}}\right), \\
0<y<\infty, \lambda_{m}>0, \quad m=1,2,3 .
\end{gathered}
$$

Table 3. MWT of a 3-CMED.

\begin{tabular}{cccc}
\hline $\boldsymbol{\lambda}_{\mathbf{1}}, \boldsymbol{\lambda}_{\mathbf{2}}, \boldsymbol{\lambda}_{\mathbf{3}}, \boldsymbol{p}_{\mathbf{1}}, \boldsymbol{p}_{\mathbf{2}}$ & $\boldsymbol{Y}=\mathbf{1}$ & $\boldsymbol{Y}=\mathbf{5}$ & $\boldsymbol{Y}=\mathbf{1 0}$ \\
\hline $2,3,4,0.1,0.2$ & 1.326540 & 5.291690 & 10.29170 \\
$2,3,4,0.2,0.3$ & 1.374520 & 5.325040 & 10.32500 \\
$2,3,4,0.3,0.4$ & 1.424020 & 5.358400 & 10.35830 \\
$2,3,4,0.4,0.5$ & 1.475120 & 5.391760 & 10.39170 \\
& & & \\
$3,2,1,0.2,0.1$ & 2.154490 & 5.839520 & 10.81700 \\
$4,3,2,0.3,0.2$ & 1.475810 & 5.391780 & 10.39170 \\
$5,4,3,0.4,0.3$ & 1.277660 & 5.255000 & 10.25500 \\
$6,5,4,0.5,0.4$ & 1.194010 & 5.188330 & 10.18830 \\
& & & \\
$3,2,1,0.4,0.2$ & 1.819170 & 5.645900 & 10.63350 \\
$5,4,3,0.4,0.2$ & 1.289110 & 5.263330 & 10.26330 \\
$7,6,5,0.4,0.2$ & 1.173980 & 5.170480 & 10.17050 \\
$9,8,7,0.4,0.2$ & 1.127060 & 5.126590 & 10.12660 \\
\hline
\end{tabular}




$$
h(y ; \Psi)=\frac{p_{1} \frac{y}{\lambda_{1}^{2}} \exp \left(-\frac{y^{2}}{2 \lambda_{1}^{2}}\right)+p_{2} \frac{y}{\lambda_{2}^{2}} \exp \left(-\frac{y^{2}}{2 \lambda_{2}^{2}}\right)+\left(1-p_{1}-p_{2}\right) \frac{y}{\lambda_{3}^{2}} \exp \left(-\frac{y^{2}}{2 \lambda_{3}^{2}}\right)}{p_{1} \exp \left(-\frac{y^{2}}{2 \lambda_{1}^{2}}\right)+p_{2} \exp \left(-\frac{y^{2}}{2 \lambda_{2}^{2}}\right)+\left(1-p_{1}-p_{2}\right) \exp \left(-\frac{y^{2}}{2 \lambda_{3}^{2}}\right)} .
$$

\section{Box V}

Table 4. Hazard rate of a 3-CMRD.

\begin{tabular}{cccc}
\hline $\boldsymbol{\lambda}_{\mathbf{1}}, \boldsymbol{\lambda}_{\mathbf{2}}, \boldsymbol{\lambda}_{\mathbf{3}}, \boldsymbol{p}_{\mathbf{1}}, \boldsymbol{p}_{\mathbf{2}}$ & $\boldsymbol{Y}=\mathbf{1}$ & $\boldsymbol{Y}=\mathbf{5}$ & $\boldsymbol{Y}=\mathbf{1 0}$ \\
\hline $12,14,16,0.1,0.2$ & 0.004449 & 0.022190 & 0.044054 \\
$12,14,16,0.2,0.3$ & 0.004872 & 0.024280 & 0.048072 \\
$12,14,16,0.3,0.4$ & 0.005295 & 0.026392 & 0.052263 \\
$12,14,16,0.4,0.5$ & 0.005719 & 0.028527 & 0.056635 \\
& & & \\
$12,10,8,0.2,0.1$ & 0.013320 & 0.065804 & 0.126239 \\
$14,12,10,0.3,0.2$ & 0.007917 & 0.039301 & 0.076806 \\
$16,14,12,0.4,0.3$ & 0.005176 & 0.025784 & 0.050983 \\
$18,16,14,0.5,0.4$ & 0.003616 & 0.018055 & 0.035965 \\
& & & \\
$10,8,6,0.5,0.3$ & 0.015220 & 0.073516 & 0.133827 \\
$12,10,8,0.5,0.3$ & 0.009592 & 0.047322 & 0.090994 \\
$14,12,10,0.5,0.3$ & 0.006633 & 0.032957 & 0.064684 \\
$16,14,12,0.5,0.3$ & 0.004872 & 0.024280 & 0.048072 \\
\hline
\end{tabular}

The failure rate function or hazard rate function for a 3-CMRD is defined as shown in Box V.

The trend of the hazard rate function (Eq. (20)) for some fixed values of component and proportion parameters is depicted in Figures 4-6. From Figures 4-6, the effects of parameters $\lambda_{1}, \lambda_{2}, \lambda_{3}, p_{1}$ and $p_{2}$ on hazard rate for the 3 -CMRD can be observed. Flexibility of hazard rate function can be seen in Figures 4-6.

Using Eq. (20), the hazard rate of a 3 -CMRD is evaluated for the parametric values fixed in Figures 46. The numerical results, so obtained, are showcased in Table 4.

From Figures 4-6 and the entries in Table 4, it is noticed that, in general, failure rate for a 3-CMRD follows an increasing trend over time. When $\lambda_{1}, \lambda_{2}, \lambda_{3}$ failure rate increases as proportion parameters increase (see row 1 of Table 4). On the other hand, when $\lambda_{1}, \lambda_{2}, \lambda_{3}$ failure rate decreases as component parameters increase (see rows 2 and 3 of Table 4). Also, there are higher chances of failure when both the component and proportion parameters are relatively smaller (see row 2 of Table 4 ).

\subsection{Cumulative hazard rate and reversed hazard rate functions for a 3-CMRD}

The cumulative hazard rate function, $H(y ; \Psi)$, and reversed hazard rate function, $r(y ; \Psi)$, for a 3 -CMRD

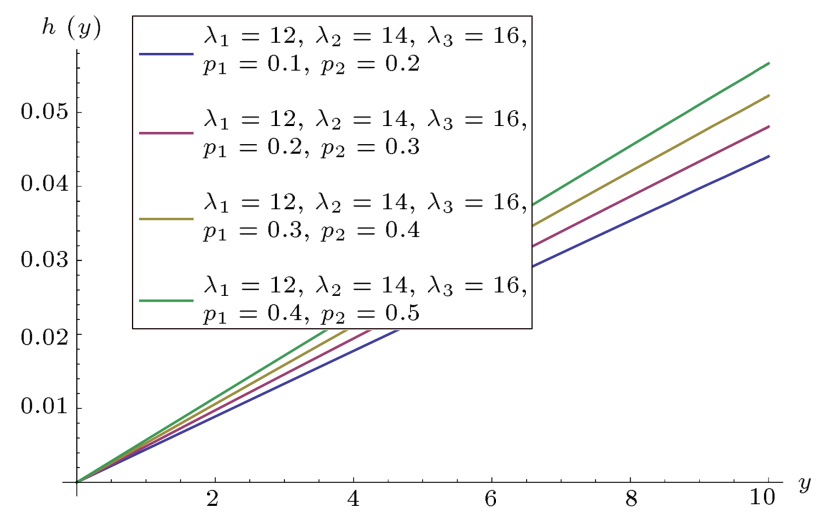

Figure 4. Graphs of hazard rate function for a 3-CMED for parameters $\left\{\left(\lambda_{1}, \lambda_{2}, \lambda_{3}, p_{1}, p_{2}\right)=(12,14,16,0.1,0.2)\right.$, $(12,14,16,0.2,0.3),(12,14,16,0.3,0.4),(12,14,16,0.4$, $0.5)\}$.

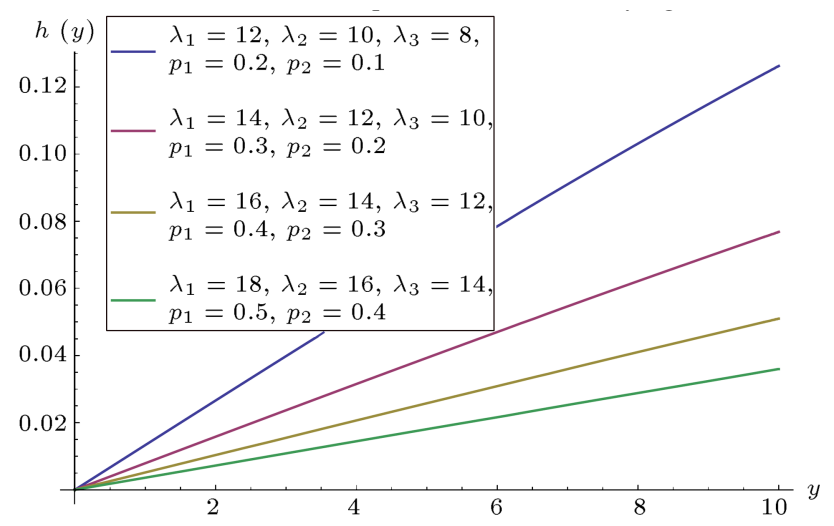

Figure 5. Graphs of hazard rate function for a 3-CMED for parameters $\left\{\left(\lambda_{1}, \lambda_{2}, \lambda_{3}, p_{1}, p_{2}\right)=(12,10,8,0.2,0.1)\right.$, $(14,12,10,0.3,0.2),(16,14,12,0.4,0.3),(18,16,14,0.5$, $0.4)\}$.

using Eqs. (5) and (6) are obtained by Eqs. (21) and (22), respectively, as shown in Box VI.

\subsection{Mean residual life and mean waiting time functions for a 3-CMRD}

The MRL function or life expectancy function for a 3CMRD is obtained by Eq. (23) as shown in Box VII. Using Eq. (23), the MRL of a 3-CMRD is evaluated for the parametric values fixed in Figures 4-6. The numerical results, so obtained, are given in Table 5 .

From the entries in Table 5 , it is seen that, in general, MRL for a 3-CMRD follows a decreasing trend 
$H(y ; \Psi)=-\ln \left\{p_{1} \exp \left(-\frac{y^{2}}{2 \lambda_{1}^{2}}\right)+p_{2} \exp \left(-\frac{y^{2}}{2 \lambda_{2}^{2}}\right)+\left(1-p_{1}-p_{2}\right) \exp \left(-\frac{y^{2}}{2 \lambda_{3}^{2}}\right)\right\}$,

and

$$
r(y ; \Psi)=\frac{p_{1} \frac{y}{\lambda_{1}^{2}} \exp \left(-\frac{y^{2}}{2 \lambda_{1}^{2}}\right)+p_{2} \frac{y}{\lambda_{2}^{2}} \exp \left(-\frac{y^{2}}{2 \lambda_{2}^{2}}\right)+\left(1-p_{1}-p_{2}\right) \frac{y}{\lambda_{3}^{2}} \exp \left(-\frac{y^{2}}{2 \lambda_{3}^{2}}\right)}{1-p_{1} \exp \left(-\frac{y^{2}}{2 \lambda_{1}^{2}}\right)-p_{2} \exp \left(-\frac{y^{2}}{2 \lambda_{2}^{2}}\right)-\left(1-p_{1}-p_{2}\right) \exp \left(-\frac{y^{2}}{2 \lambda_{3}^{2}}\right)} .
$$

\section{Box VI}

$$
m(y ; \Psi)=\frac{p_{1} \lambda_{1} \sqrt{\frac{\pi}{2}}\left\{1-\operatorname{Erf}\left(\frac{y}{\sqrt{2} \lambda_{1}}\right)\right\}+p_{2} \lambda_{2} \sqrt{\frac{\pi}{2}}\left\{1-\operatorname{Erf}\left(\frac{y}{\sqrt{2} \lambda_{2}}\right)\right\}+\left(1-p_{1}-p_{2}\right) \lambda_{3} \sqrt{\frac{\pi}{2}}\left\{1-\operatorname{Erf}\left(\frac{y}{\sqrt{2} \lambda_{3}}\right)\right\}}{p_{1} \exp \left(-\frac{y^{2}}{2 \lambda_{1}^{2}}\right)+p_{2} \exp \left(-\frac{y^{2}}{2 \lambda_{2}^{2}}\right)+\left(1-p_{1}-p_{2}\right) \exp \left(-\frac{y^{2}}{2 \lambda_{3}^{2}}\right)} .
$$

\section{Box VII}

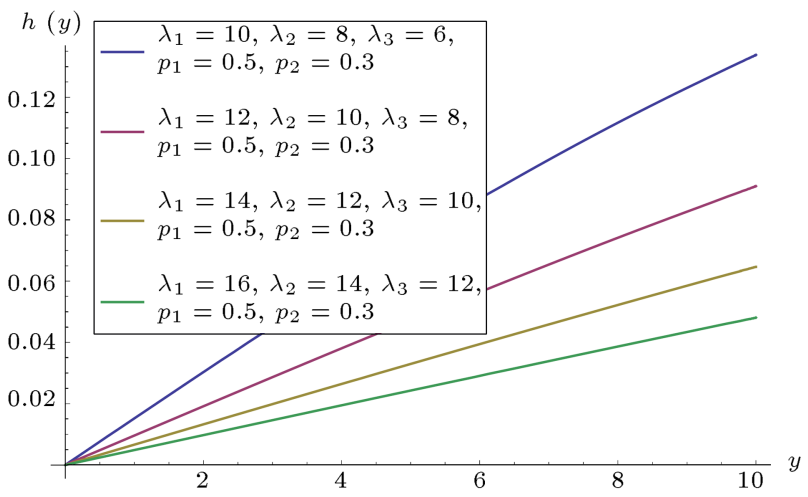

Figure 6. Graphs of hazard rate function for a 3-CMED for parameters $\left\{\left(\lambda_{1}, \lambda_{2}, \lambda_{3}, p_{1}, p_{2}\right)=(10,8,6,0.5,0.3)\right.$, $(12,10,8,0.5,0.3),(14,12,10,0.5,0.3),(16,14,12,0.5$, $0.3)\}$.

over time. When $\lambda_{1}<\lambda_{2}<\lambda_{3}$, MRL decreases as proportion parameters increase (see row 1 of Table 5 ). On the other hand, when $\lambda_{1}>\lambda_{2}>\lambda_{3}$, MRL increases as component parameters increase (see rows 2 and 3 of Table 5). Using Eq. (8), the MWT function for a 3CMRD is obtained by Eq. (24) as shown in Box VIII.
Table 5. MRL of a 3-CMRD.

\begin{tabular}{cccc}
\hline $\boldsymbol{\lambda}_{\mathbf{1}}, \boldsymbol{\lambda}_{\mathbf{2}}, \boldsymbol{\lambda}_{\mathbf{3}}, \boldsymbol{p}_{\mathbf{1}}, \boldsymbol{p}_{\mathbf{2}}$ & $\boldsymbol{Y}=\mathbf{1}$ & $\boldsymbol{Y}=\mathbf{5}$ & $\boldsymbol{Y}=\mathbf{1 0}$ \\
\hline $12,14,16,0.1,0.2$ & 18.09130 & 14.94920 & 12.15690 \\
$12,14,16,0.2,0.3$ & 17.34140 & 14.23790 & 11.52970 \\
$12,14,16,0.3,0.4$ & 16.59120 & 13.51910 & 10.87580 \\
$12,14,16,0.4,0.5$ & 15.84060 & 12.79260 & 10.19340 \\
& & & \\
$12,10,8,0.2,0.1$ & 10.35080 & 7.721280 & 5.929370 \\
$14,12,10,0.3,0.2$ & 13.59350 & 10.70350 & 8.428180 \\
$16,14,12,0.4,0.3$ & 16.84150 & 13.76350 & 11.11020 \\
$18,16,14,0.5,0.4$ & 20.09260 & 16.87520 & 13.92330 \\
& & & \\
$10,8,6,0.5,0.3$ & 9.855810 & 7.325830 & 5.642930 \\
$12,10,8,0.5,0.3$ & 12.34580 & 9.549830 & 7.433610 \\
$14,12,10,0.5,0.3$ & 14.84200 & 11.86790 & 9.419580 \\
$16,14,12,0.5,0.3$ & 17.34140 & 14.23790 & 11.52970 \\
\hline
\end{tabular}

Using Eq. (24), the MWT of a 3-CMRD is evaluated for the parametric values fixed in Figures 4-6. The numerical results, so obtained, are presented in Table 6 .

From the entries in Table 6 , it is obvious that, in general, MWT for a 3-CMRD follows an increasing trend over time. When $\lambda_{1}<\lambda_{2}<\lambda_{3}$, MWT increases as

$$
\mu(y ; \Psi)=\frac{y-p_{1} \lambda_{1} \sqrt{\frac{\pi}{2}} \operatorname{Erf}\left(\frac{y}{\sqrt{2} \lambda_{1}}\right)-p_{2} \lambda_{2} \sqrt{\frac{\pi}{2}} \operatorname{Erf}\left(\frac{y}{\sqrt{2} \lambda_{2}}\right)-\left(1-p_{1}-p_{2}\right) \lambda_{3} \sqrt{\frac{\pi}{2}} \operatorname{Erf}\left(\frac{y}{\sqrt{2} \lambda_{3}}\right)}{1-p_{1} \exp \left(-\frac{y^{2}}{2 \lambda_{1}^{2}}\right)-p_{2} \exp \left(-\frac{y^{2}}{2 \lambda_{2}^{2}}\right)-\left(1-p_{1}-p_{2}\right) \exp \left(-\frac{y^{2}}{2 \lambda_{3}^{2}}\right)} .
$$


Table 6. MWT of a 3-CMRD.

\begin{tabular}{cccc}
\hline $\boldsymbol{\lambda}_{\mathbf{1}}, \boldsymbol{\lambda}_{\mathbf{2}}, \boldsymbol{\lambda}_{\mathbf{3}}, \boldsymbol{p}_{\mathbf{1}}, \boldsymbol{p}_{\mathbf{2}}$ & $\boldsymbol{Y}=\mathbf{1}$ & $\boldsymbol{Y}=\mathbf{5}$ & $\boldsymbol{Y}=\mathbf{1 0}$ \\
\hline $12,14,16,0.1,0.2$ & 0.333488 & 1.686060 & 3.488280 \\
$12,14,16,0.2,0.3$ & 0.333505 & 1.688110 & 3.504600 \\
$12,14,16,0.3,0.4$ & 0.333519 & 1.689840 & 3.518610 \\
$12,14,16,0.4,0.5$ & 0.333531 & 1.691330 & 3.530760 \\
& & & \\
$12,10,8,0.2,0.1$ & 0.333810 & 1.726250 & 3.807210 \\
$14,12,10,0.3,0.2$ & 0.333617 & 1.702140 & 3.616060 \\
$16,14,12,0.4,0.3$ & 0.333516 & 1.689510 & 3.515760 \\
$18,16,14,0.5,0.4$ & 0.333458 & 1.682190 & 3.457630 \\
& & & \\
$10,8,6,0.5,0.3$ & 0.333940 & 1.741480 & 3.904260 \\
$12,10,8,0.5,0.3$ & 0.333691 & 1.711170 & 3.683860 \\
$14,12,10,0.5,0.3$ & 0.333572 & 1.696450 & 3.570310 \\
$16,14,12,0.5,0.3$ & 0.333505 & 1.688110 & 3.504600 \\
\hline
\end{tabular}

proportion parameters increase (see row 1 of Table 6 ). On the other hand, when $\lambda_{1}>\lambda_{2}>\lambda_{3}$, MWT decreases as component parameters increase (see rows 2 and 3 of Table 6).

\section{Reliability properties of a three-component mixture of Pareto distributions}

Using Eqs. (1) and (2), a finite three-Component Mixture of Pareto Distributions (3-CMPD) with mixing proportions $p_{1}$ and $p_{2}$ has the pdf and cdf as follows:

$$
\begin{aligned}
& f(y ; \Psi)=p_{1} \lambda_{1} y^{-\left(\lambda_{1}+1\right)}+p_{2} \lambda_{2} y^{-\left(\lambda_{2}+1\right)} \\
& +\left(1-p_{1}-p_{2}\right) \lambda_{3} y^{-\left(\lambda_{3}+1\right)}, \\
& 1<y<\infty, \quad \lambda_{m}>0, \quad m=1,2,3 \\
& F(y ; \Psi)=1-p_{1} y^{-\lambda_{1}}-p_{2} y^{-\lambda_{2}}-\left(1-p_{1}-p_{2}\right) y^{-\lambda_{3}} .
\end{aligned}
$$

\subsection{Reliability and failure rate functions for a 3-CMPD}

The reliability function or survival function for a 3CMPD is written as follows:

$$
\begin{aligned}
& R(y ; \Psi)=p_{1} y^{-\lambda_{1}}+p_{2} y^{-\lambda_{2}}+\left(1-p_{1}-p_{2}\right) y^{-\lambda_{3}}, \\
& 1<y<\infty, \quad \lambda_{m}>0, \quad m=1,2,3 .
\end{aligned}
$$

The failure rate function or hazard rate function for a 3-CMPD is defined as follows:

$$
\begin{aligned}
& h(y ; \Psi)= \\
& \frac{p_{1} \lambda_{1} y^{-\left(\lambda_{1}+1\right)}+p_{2} \lambda_{2} y^{-\left(\lambda_{2}+1\right)}+\left(1-p_{1}-p_{2}\right) \lambda_{3} y^{-\left(\lambda_{3}+1\right)}}{p_{1} y^{-\lambda_{1}}+p_{2} y^{-\lambda_{2}}+\left(1-p_{1}-p_{2}\right) y^{-\lambda_{3}}} .
\end{aligned}
$$

The trend of the hazard rate Function (Eq. (28)) for some values of component and proportion parameters is shown in Figures 7-9. From Figures 7-9, the

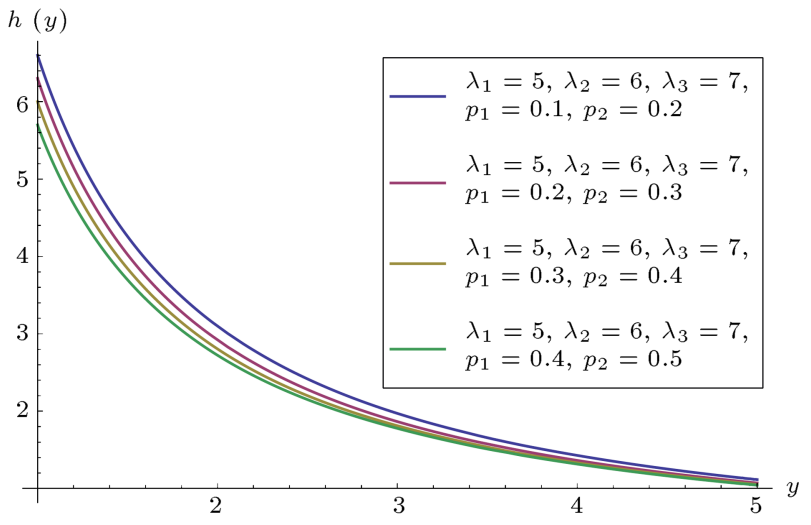

Figure 7. Graphs of hazard rate function for a 3-CMED for parameters $\left\{\left(\lambda_{1}, \lambda_{2}, \lambda_{3}, p_{1}, p_{2}\right)=(5,6,7,0.1,0.2),(5\right.$, $6,7,0.2,0.3),(5,6,7,0.3,0.4),(5,6,7,0.4,0.5)\}$.

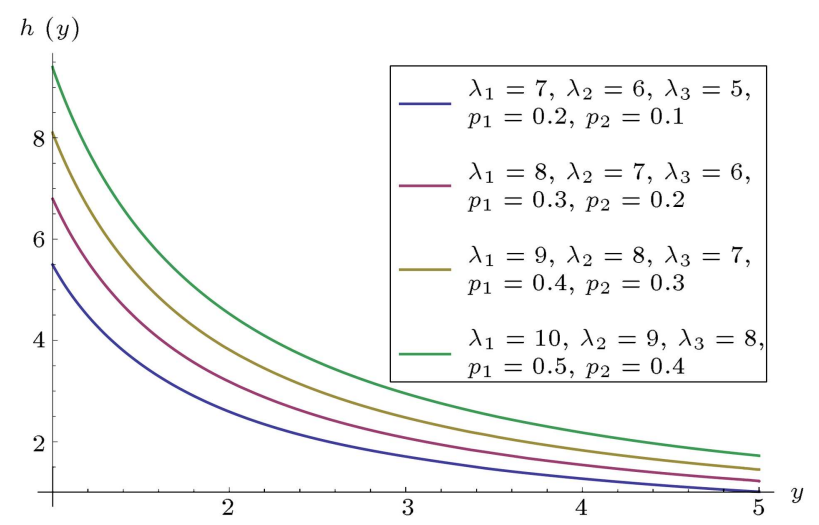

Figure 8. Graphs of hazard rate function for a 3-CMED for parameters $\left\{\left(\lambda_{1}, \lambda_{2}, \lambda_{3}, p_{1}, p_{2}\right)=(7,6,5,0.2,0.1),(8\right.$, $7,6,0.3,0.2),(9,8,7,0.4,0.3),(10,9,8,0.5,0.4)\}$.

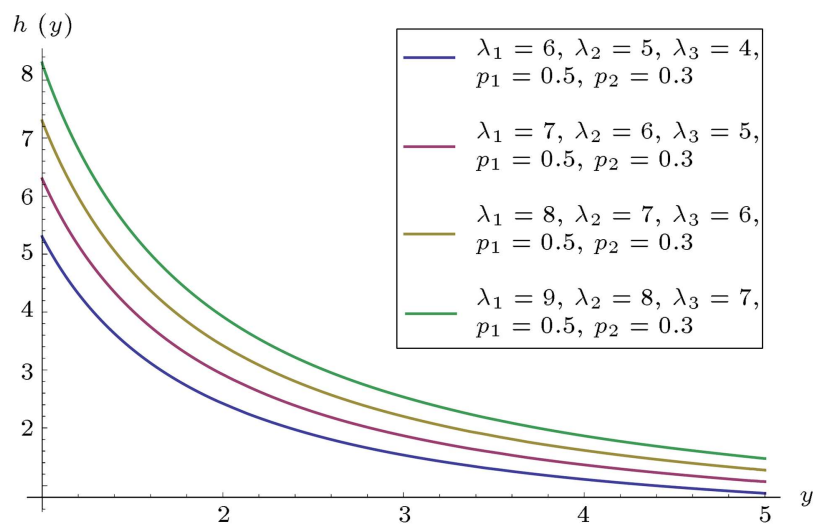

Figure 9. Graphs of hazard rate function for a 3-CMED for parameters $\left\{\left(\lambda_{1}, \lambda_{2}, \lambda_{3}, p_{1}, p_{2}\right)=(6,5,4,0.5,0.3),(7\right.$, $6,5,0.5,0.3),(8,7,6,0.5,0.3),(9,8,7,0.5,0.3)\}$.

effects of parameters $\lambda_{1}, \lambda_{2}, \lambda_{3}$ and $p_{2}$ on hazard rate for the 3-CMPD can be observed. These graphs also explain the flexibility of the hazard rate for a 3-CMPD.

Using Eq. (28), the hazard rate of a 3 -CMPD is evaluated for the parametric values fixed in Figures 7- 
Table 7. Hazard rate of a 3-CMPD.

\begin{tabular}{rccc}
\hline $\boldsymbol{\lambda}_{\mathbf{1}}, \boldsymbol{\lambda}_{\mathbf{2}}, \boldsymbol{\lambda}_{\mathbf{3}}, \boldsymbol{p}_{\mathbf{1}}, \boldsymbol{p}_{\mathbf{2}}$ & $\boldsymbol{Y}=\mathbf{1}$ & $\boldsymbol{Y}=\mathbf{5}$ & $\boldsymbol{Y}=\mathbf{1 0}$ \\
\hline $5,6,7,0.1,0.2$ & 6.600000 & 1.114290 & 0.526772 \\
$5,6,7,0.2,0.3$ & 6.300000 & 1.071430 & 0.517021 \\
$5,6,7,0.3,0.4$ & 6.000000 & 1.053060 & 0.513411 \\
$5,6,7,0.4,0.5$ & 5.700000 & 1.042860 & 0.168017 \\
& & & \\
$7,6,5,0.2,0.1$ & 5.500000 & 1.009890 & 0.501966 \\
$8,7,6,0.3,0.2$ & 6.800000 & 1.223190 & 0.604971 \\
$9,8,7,0.4,0.3$ & 8.100000 & 1.448940 & 0.711377 \\
$10,9,8,0.5,0.4$ & 9.400000 & 1.720000 & 0.834483 \\
& & & \\
$6,5,4,0.5,0.3$ & 5.300000 & 0.871429 & 0.417021 \\
$7,6,5,0.5,0.3$ & 6.300000 & 1.071430 & 0.517021 \\
$8,7,6,0.5,0.3$ & 7.300000 & 1.271430 & 0.617021 \\
$9,8,7,0.5,0.3$ & 8.300000 & 1.471430 & 0.717021 \\
\hline
\end{tabular}

9. The numerical results, so obtained, are showcased in Table 7.

From Figures 7-9 and the entries in Table 7, it is observed that, in general, failure rate for a 3 CMPD follows a decreasing trend over time. When $\lambda_{1}<\lambda_{2}<\lambda_{3}$, failure rate decreases as proportion parameters increase (see row 1 of Table 7). On the other hand, when $\lambda_{1}>\lambda_{2}>\lambda_{3}$, failure rate increases as component parameters increase (see rows 2 and 3 of Table 7). Also, there are higher chances of failure when both the component and proportion parameters are relatively larger (see row 2 of Table 7 ).

\subsection{Cumulative hazard rate and reversed hazard rate functions for a 3-CMPD}

The cumulative hazard rate function, $H(y ; \Psi)$, and reversed hazard rate function, $r(y ; \Psi)$, for a 3 -CMPD using Eqs. (5) and (6) are obtained by Eqs. (29) and (30), respectively, as shown in Box IX.

\subsection{Mean residual life and mean waiting time functions for a 3-CMPD}

The MRL function or life expectancy function for a 3-CMPD is:
Table 8. MRL of a 3-CMPD.

\begin{tabular}{rccc}
\hline $\boldsymbol{\lambda}_{\mathbf{1}}, \boldsymbol{\lambda}_{\mathbf{2}}, \boldsymbol{\lambda}_{\mathbf{3}}, \boldsymbol{p}_{\mathbf{1}}, \boldsymbol{p}_{\mathbf{2}}$ & $\boldsymbol{Y}=\mathbf{1}$ & $\boldsymbol{Y}=\mathbf{5}$ & $\boldsymbol{Y}=\mathbf{1 0}$ \\
\hline $5,6,7,0.1,0.2$ & 0.181667 & 1.121030 & 2.375330 \\
$5,6,7,0.2,0.3$ & 0.193333 & 1.166670 & 2.418440 \\
$5,6,7,0.3,0.4$ & 0.205000 & 1.186220 & 2.434400 \\
$5,6,7,0.4,0.5$ & 0.216667 & 1.197090 & 2.442720 \\
& & & \\
$7,6,5,0.2,0.1$ & 0.228333 & 1.238550 & 2.490640 \\
$8,7,6,0.3,0.2$ & 0.176190 & 0.981712 & 1.983980 \\
$9,8,7,0.4,0.3$ & 0.142857 & 0.805471 & 1.640290 \\
$10,9,8,0.5,0.4$ & 0.119841 & 0.662698 & 1.368360 \\
& & & \\
$6,5,4,0.5,0.3$ & 0.241667 & 1.529760 & 3.198580 \\
$7,6,5,0.5,0.3$ & 0.193333 & 1.166670 & 2.418440 \\
$8,7,6,0.5,0.3$ & 0.161429 & 0.943878 & 1.945290 \\
$9,8,7,0.5,0.3$ & 0.138690 & 0.792942 & 1.627410 \\
\hline
\end{tabular}

$$
\begin{aligned}
& m(y ; \Psi)= \\
& \frac{\frac{p_{1}}{\left(\lambda_{1}-1\right)} y^{1-\lambda_{1}}+\frac{p_{2}}{\left(\lambda_{2}-1\right)} y^{1-\lambda_{2}}+\frac{\left(1-p_{1}-p_{2}\right)}{\left(\lambda_{3}-1\right)} y^{1-\lambda_{3}}}{p_{1} y^{-\lambda_{1}}+p_{2} y^{-\lambda_{2}}+\left(1-p_{1}-p_{2}\right) y^{-\lambda_{3}}} .
\end{aligned}
$$

Using Eq. (31), the MRL of a $3-\mathrm{CMPD}$ is evaluated for the parametric values fixed in Figures 7-9. The numerical results, so obtained, are presented in Table 8.

From the entries available in Table 8 , it is obvious that, in general, MRL for a 3-CMPD follows an increasing trend over time. When $\lambda_{1}<\lambda_{2}<\lambda_{3}$, MRL increases as proportion parameters increase (see row 1 of Table 8). On the other hand, when $\lambda_{1}>\lambda_{2}>\lambda_{3}$, MRL decreases as component parameters increase (see rows 2 and 3 of Table 8). Using Eq. (8), the MWT function for a 3-CMPD is obtained by Eq. (32) as shown in Box X.

Using the above Expression (32), the MWT of a $3-C M P D$ is evaluated for the parametric values fixed in Figures 7-9. The numerical results, so obtained, are given in Table 9 .

From the entries in Table 9, it is seen that, in general, MWT for a 3-CMPD follows an increasing trend over time. When $\lambda_{1}<\lambda_{2}<\lambda_{3}$, MWT increases as

$$
H(y ; \Psi)=-\ln \left\{p_{1} y^{-\lambda_{1}}+p_{2} y^{-\lambda_{2}}+\left(1-p_{1}-p_{2}\right) y^{-\lambda_{3}}\right\}
$$

and

$$
r(y ; \Psi)=\frac{p_{1} \lambda_{1} y^{-\left(\lambda_{1}+1\right)}+p_{2} \lambda_{2} y^{-\left(\lambda_{2}+1\right)}+\left(1-p_{1}-p_{2}\right) \lambda_{3} y^{-\left(\lambda_{3}+1\right)}}{1-p_{1} y^{-\lambda_{1}}-p_{2} y^{-\lambda_{2}}-\left(1-p_{1}-p_{2}\right) y^{-\lambda_{3}}} .
$$




$$
\bar{\mu}(y ; \Psi)=\frac{y-\frac{p_{1}}{\left(1-\lambda_{1}\right)}\left\{y^{1-\lambda_{1}}-\lambda_{1}\right\}-\frac{p_{2}}{\left(1-\lambda_{2}\right)}\left\{y^{1-\lambda_{2}}-\lambda_{2}\right\}-\frac{\left(1-p_{1}-p_{2}\right)}{\left(1-\lambda_{3}\right)}\left\{y^{1-\lambda_{3}}-\lambda_{3}\right\}}{1-p_{1} y^{-\lambda_{1}}-p_{2} y^{-\lambda_{2}}-\left(1-p_{1}-p_{2}\right) y^{-\lambda_{3}}} .
$$

Box X

Table 9. MWT of a 3-CMPD.

\begin{tabular}{cccc}
\hline $\boldsymbol{\lambda}_{\mathbf{1}}, \boldsymbol{\lambda}_{\mathbf{2}}, \boldsymbol{\lambda}_{\mathbf{3}}, \boldsymbol{p}_{\mathbf{1}}, \boldsymbol{p}_{\mathbf{2}}$ & $\boldsymbol{Y}=\mathbf{1}$ & $\boldsymbol{Y}=\mathbf{5}$ & $\boldsymbol{Y}=\mathbf{1 0}$ \\
\hline $5,6,7,0.1,0.2$ & 2.866750 & 6.181940 & 11.18170 \\
$5,6,7,0.2,0.3$ & 2.906390 & 6.193780 & 11.19340 \\
$5,6,7,0.3,0.4$ & 2.946910 & 6.205630 & 11.20500 \\
$5,6,7,0.4,0.5$ & 2.988360 & 6.217480 & 11.21670 \\
& & & \\
$7,6,5,0.2,0.1$ & 3.029530 & 6.229500 & 11.22840 \\
$8,7,6,0.3,0.2$ & 2.849370 & 6.176370 & 11.17620 \\
$9,8,7,0.4,0.3$ & 2.742810 & 6.142880 & 11.14290 \\
$10,9,8,0.5,0.4$ & 2.677120 & 6.119840 & 11.11980 \\
& & & \\
$6,5,4,0.5,0.3$ & 3.071430 & 6.243780 & 11.24190 \\
$7,6,5,0.5,0.3$ & 2.906390 & 6.193780 & 11.19340 \\
$8,7,6,0.5,0.3$ & 2.800570 & 6.161520 & 11.16140 \\
$9,8,7,0.5,0.3$ & 2.730250 & 6.138710 & 11.13870 \\
\hline
\end{tabular}

proportion parameters increase (see row 1 of Table 9 ). On the other hand, when $\lambda_{1}>\lambda_{2}>\lambda_{3}$, MWT decreases as component parameters increase (see rows 2 and 3 of Table 9).

\section{Reliability properties of a three-component mixture of Burr type-XII distributions}

Using Eqs. (1) and (2), a finite three-component mixture of Burr Type-XII distributions (3-CMBD) with mixing proportions $p_{1}$ and $p_{2}$ has the pdf and cdf as follows:

$$
\begin{aligned}
f(y ; \Psi)= & p_{1} \lambda_{1}(1+y)^{-\left(\lambda_{1}+1\right)}+p_{2} \lambda_{2}(1+y)^{-\left(\lambda_{2}+1\right)} \\
& +\left(1-p_{1}-p_{2}\right) \lambda_{3}(1+y)^{-\left(\lambda_{3}+1\right)} \\
0<y< & \infty, \quad \lambda_{m}>0, \quad m=1,2,3 \\
F(y ; \Psi)= & 1-p_{1}(1+y)^{-\lambda_{1}}-p_{2}(1+y)^{-\lambda_{2}} \\
& -\left(1-p_{1}-p_{2}\right)(1+y)^{-\lambda_{3}} .
\end{aligned}
$$

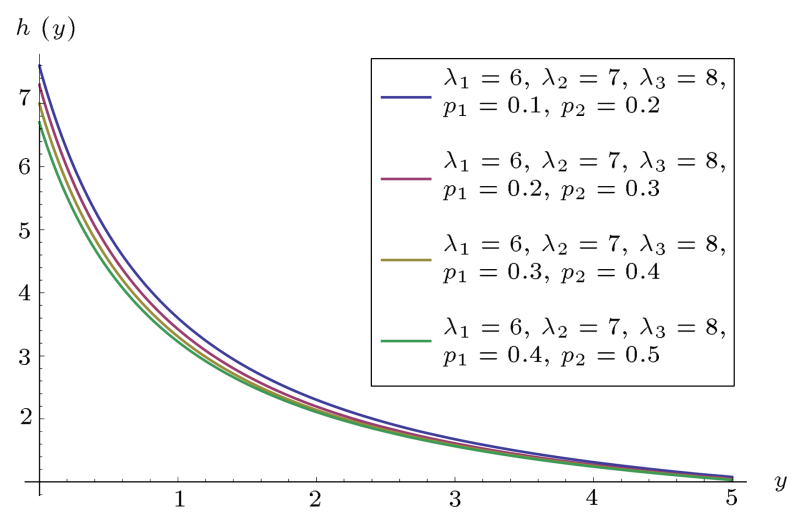

Figure 10. Graphs of hazard rate function for a 3-CMED for parameters $\left\{\left(\lambda_{1}, \lambda_{2}, \lambda_{3}, p_{1}, p_{2}\right)=(6,7,8,0.1,0.2),(6\right.$, $7,8,0.2,0.3),(6,7,8,0.3,0.4),(6,7,8,0.4,0.5)\}$.

\subsection{Reliability and failure rate functions for a 3- $C M B D$}

The reliability function or survival function for a 3CMBD is written as:

$$
\begin{aligned}
& R(y ; \Psi)= p_{1}(1+y)^{-\lambda_{1}}+p_{2}(1+y)^{-\lambda_{2}} \\
&+\left(1-p_{1}-p_{2}\right)(1+y)^{-\lambda_{3}} \\
& 0<y<\infty, \quad \lambda_{m}>0, \quad m=1,2,3 .
\end{aligned}
$$

The failure rate function or hazard rate function for a 3-CMBD is defined by Eq. (36) as shown in Box XI.

The trend of the hazard rate Function (Eq. (36)) for some fixed values of component and proportion parameters is illustrated in Figures 10-12. From Figures 10-12, the effects of parameters $\lambda_{1}, \lambda_{2}, \lambda_{3}, p_{1}$ and $p_{2}$ on hazard rate for the 3 -CMBD can be observed.

Using Eq. (36), the hazard rate of a 3-CMBD is evaluated for the parametric values fixed in Figures 1012. The numerical results, so obtained, are presented in Table 10.

From Figures 10-12 and the entries in Table 10, it is seen that, in general, failure rate for a 3-CMED

$$
h(y ; \Psi)=\frac{p_{1} \lambda_{1}(1+y)^{-\left(\lambda_{1}+1\right)}+p_{2} \lambda_{2}(1+y)^{-\left(\lambda_{2}+1\right)}+\left(1-p_{1}-p_{2}\right) \lambda_{3}(1+y)^{-\left(\lambda_{3}+1\right)}}{p_{1}(1+y)^{-\lambda_{1}}+p_{2}(1+y)^{-\lambda_{2}}+\left(1-p_{1}-p_{2}\right)(1+y)^{-\lambda_{3}}} .
$$




$$
\begin{aligned}
& H(y ; \Psi)=-\ln \left\{p_{1}(1+y)^{-\lambda_{1}}+p_{2}(1+y)^{-\lambda_{2}}+\left(1-p_{1}-p_{2}\right)(1+y)^{-\lambda_{3}}\right\}, \\
& r(y ; \Psi)=\frac{p_{1} \lambda_{1}(1+y)^{-\left(\lambda_{1}+1\right)}+p_{2} \lambda_{2}(1+y)^{-\left(\lambda_{2}+1\right)}+\left(1-p_{1}-p_{2}\right) \lambda_{3}(1+y)^{-\left(\lambda_{3}+1\right)}}{1-p_{1}(1+y)^{-\lambda_{1}}-p_{2}(1+y)^{-\lambda_{2}}-\left(1-p_{1}-p_{2}\right)(1+y)^{-\lambda_{3}}} .
\end{aligned}
$$

\section{Box XII}

$$
m(y ; \Psi)=\frac{\frac{p_{1}}{\left(\lambda_{1}-1\right)}(1+y)^{1-\lambda_{1}}+\frac{p_{2}}{\left(\lambda_{2}-1\right)}(1+y)^{1-\lambda_{2}}+\frac{\left(1-p_{1}-p_{2}\right)}{\left(\lambda_{3}-1\right)}(1+y)^{1-\lambda_{3}}}{p_{1}(1+y)^{-\lambda_{1}}+p_{2}(1+y)^{-\lambda_{2}}+\left(1-p_{1}-p_{2}\right)(1+y)^{-\lambda_{3}}} .
$$

\section{Box XIII}

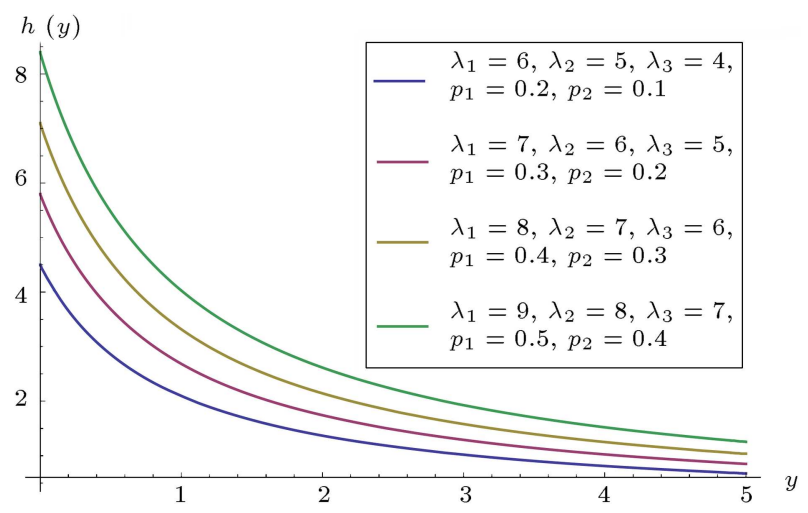

Figure 11. Graphs of hazard rate function for a 3-CMED for parameters $\left\{\left(\lambda_{1}, \lambda_{2}, \lambda_{3}, p_{1}, p_{2}\right)=(6,5,4,0.2,0.1),(7\right.$, $6,5,0.3,0.2),(8,7,6,0.4,0.3),(9,8,7,0.5,0.4)\}$.

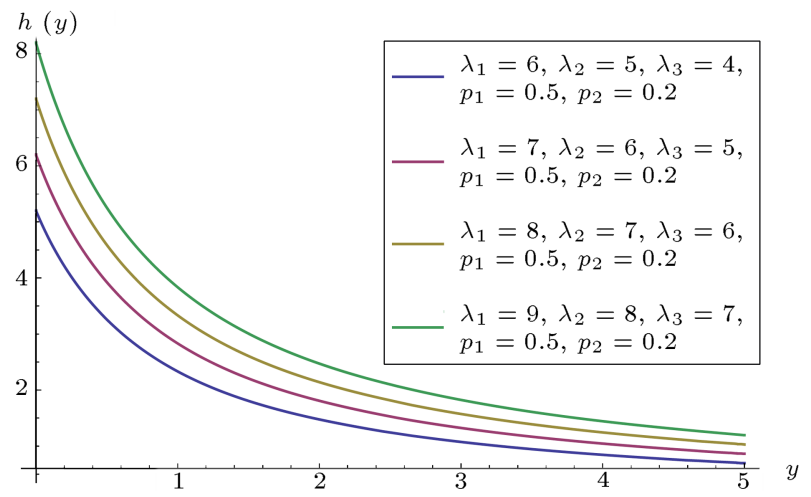

Figure 12. Graphs of hazard rate function for a 3-CMED for parameters $\left\{\left(\lambda_{1}, \lambda_{2}, \lambda_{3}, p_{1}, p_{2}\right)=(6,5,4,0.5,0.2),(7\right.$, $6,5,0.5,0.2),(8,7,6,0.5,0.2),(9,8,7,0.5,0.2)\}$.

follows a decreasing trend over time. When $\lambda_{1}<\lambda_{2}<$ $\lambda_{3}$, failure rate decreases as proportion parameters increase (see row 1 of Table 10). On the other hand, when $\lambda_{1}>\lambda_{2}>\lambda_{3}$, failure rate increases as component parameters increase (see rows 2 and 3 of Table 10). Also, there are higher chances of failure when both the
Table 10. Hazard rate of a 3-CMBD.

\begin{tabular}{cccc}
\hline $\boldsymbol{\lambda}_{\mathbf{1}}, \boldsymbol{\lambda}_{\mathbf{2}}, \boldsymbol{\lambda}_{\mathbf{3}}, \boldsymbol{p}_{\mathbf{1}}, \boldsymbol{p}_{\mathbf{2}}$ & $\boldsymbol{Y}=\mathbf{1}$ & $\boldsymbol{Y}=\mathbf{5}$ & $\boldsymbol{Y}=\mathbf{1 0}$ \\
\hline $6,7,8,0.1,0.2$ & 3.600000 & 1.078790 & 0.567273 \\
$6,7,8,0.2,0.3$ & 3.421050 & 1.049120 & 0.559416 \\
$6,7,8,0.3,0.4$ & 3.304350 & 1.037040 & 0.556541 \\
$6,7,8,0.4,0.5$ & 3.222220 & 1.030480 & 0.555051 \\
& & & \\
$6,5,4,0.2,0.1$ & 2.093750 & 0.673077 & 0.365222 \\
$7,6,5,0.3,0.2$ & 2.685190 & 0.848718 & 0.458586 \\
$8,7,6,0.4,0.3$ & 3.318180 & 1.033330 & 0.554773 \\
$9,8,7,0.5,0.4$ & 4.029410 & 1.253850 & 0.665241 \\
& & & \\
$6,5,4,0.5,0.2$ & 2.333330 & 0.696000 & 0.371096 \\
$7,6,5,0.5,0.2$ & 2.833330 & 0.862667 & 0.462005 \\
$8,7,6,0.5,0.2$ & 3.333330 & 1.029330 & 0.552914 \\
$9,8,7,0.5,0.2$ & 3.833330 & 1.196000 & 0.643823 \\
\hline
\end{tabular}

component and proportion parameters are relatively larger (see row 2 of Table 10).

\subsection{Cumulative hazard rate and reversed hazard rate functions for a 3-CMBD}

The cumulative hazard rate function, $H(y ; \Psi)$, and reversed hazard rate function, $r(y ; \Psi)$, for a 3 -CMBD using Eq. (5) and (6) are obtained by Eqs. (37) and (38), respectively, as shown in Box XII.

\subsection{Mean residual life and mean waiting time functions for a 3-CMBD}

The MRL function or life expectancy function for a 3CMBD is obtained by Eq. (39) as shown in Box XIII. Using Eq. (39), the MRL of a $3-\mathrm{CMBD}$ is evaluated for the parametric values fixed in Figures 10-12. The numerical results, so obtained, are given in Table 11.

From the entries available in Table 11, it is observed that, in general, MRL for a 3-CMBD follows an increasing trend over time. When $\lambda_{1}<\lambda_{2}<\lambda_{3}$, 


$$
\bar{\mu}(y ; \Psi)=\frac{y-\frac{p_{1}}{\left(1-\lambda_{1}\right)}\left\{(1+y)^{1-\lambda_{1}}-1\right\}-\frac{p_{2}}{\left(1-\lambda_{2}\right)}\left\{(1+y)^{1-\lambda_{2}}-1\right\}-\frac{\left(1-p_{1}-p_{2}\right)}{\left(1-\lambda_{3}\right)}\left\{(1+y)^{1-\lambda_{3}}-1\right\}}{1-p_{1}(1+y)^{-\lambda_{1}}-p_{2}(1+y)^{-\lambda_{2}}-\left(1-p_{1}-p_{2}\right)(1+y)^{-\lambda_{3}}} .
$$

Table 11. MRL of a 3-CMBD.

\begin{tabular}{cccc}
\hline $\boldsymbol{\lambda}_{\mathbf{1}}, \boldsymbol{\lambda}_{\mathbf{2}}, \boldsymbol{\lambda}_{\mathbf{3}}, \boldsymbol{p}_{\mathbf{1}}, \boldsymbol{p}_{\mathbf{2}}$ & $\boldsymbol{Y}=\mathbf{1}$ & $\boldsymbol{Y}=\mathbf{5}$ & $\boldsymbol{Y}=\mathbf{1 0}$ \\
\hline $6,7,8,0.1,0.2$ & 0.328889 & 1.112730 & 2.116890 \\
$6,7,8,0.2,0.3$ & 0.348872 & 1.144060 & 2.145560 \\
$6,7,8,0.3,0.4$ & 0.361905 & 1.156830 & 2.156050 \\
$6,7,8,0.4,0.5$ & 0.371076 & 1.163760 & 2.161490 \\
& & & \\
$6,5,4,0.2,0.1$ & 0.639583 & 1.982310 & 3.651530 \\
$7,6,5,0.3,0.2$ & 0.466667 & 1.473850 & 2.726430 \\
$8,7,6,0.4,0.3$ & 0.361039 & 1.161760 & 2.163460 \\
$9,8,7,0.5,0.4$ & 0.286415 & 0.928022 & 1.752070 \\
& & & \\
$6,5,4,0.5,0.2$ & 0.571429 & 1.920000 & 3.596150 \\
$7,6,5,0.5,0.2$ & 0.441270 & 1.451200 & 2.707220 \\
$8,7,6,0.5,0.2$ & 0.360091 & 1.167090 & 2.171260 \\
$9,8,7,0.5,0.2$ & 0.304422 & 0.976286 & 1.812680 \\
\hline
\end{tabular}

MRL increases as proportion parameters increase (see row 1 of Table 11). On the other hand, when $\lambda_{1}>\lambda_{2}>$ $\lambda_{3}$, MRL decreases as component parameters increase (see rows 2 and 3 of Table 11).

Using Eq. (8), the MWT function for a 3-CMBD is obtained by Eq. (40) as shown in Box XIV.

Using the above expression (40), the MWT of a 3-CMBD is evaluated for the parametric values fixed in Figures 10-12. The numerical results, so obtained, are showcased in Table 12.

From the entries in Table 12, it is obvious that, in general, MWT for a 3-CMBD follows an increasing trend over time. When $\lambda_{1}<\lambda_{2}<\lambda_{3}$, MWT increases as proportion parameters increase (see row 1 of Table 12). On the other hand, when $\lambda_{1}>\lambda_{2}>\lambda_{3}$, MWT decreases as component parameters increase (see rows 2 and 3 of Table 12).

\section{Concluding remarks}

The reliability analyses of three-component mixture of Exponential, Rayleigh, Pareto, and Burr Type-XII distributions are performed. It is observed that, in general, failure rate functions of 3-CMED, 3-CMPD, and 3-CMBD (3-CMRD) follow a decreasing trend (increasing) over time. Moreover, when $\lambda_{1}<\lambda_{2}<\lambda_{3}$, hazard rates of 3-CMED, 3-CMPD, and 3-CMBD (3-
Table 12. MWT of a 3 -CMBD.

\begin{tabular}{cccc}
\hline $\boldsymbol{\lambda}_{\mathbf{1}}, \boldsymbol{\lambda}_{\mathbf{2}}, \boldsymbol{\lambda}_{\mathbf{3}}, \boldsymbol{p}_{\mathbf{1}}, \boldsymbol{p}_{\mathbf{2}}$ & $\boldsymbol{Y}=\mathbf{1}$ & $\boldsymbol{Y}=\mathbf{5}$ & $\boldsymbol{Y}=\mathbf{1 0}$ \\
\hline $6,7,8,0.1,0.2$ & 1.158190 & 5.153350 & 10.15330 \\
$6,7,8,0.2,0.3$ & 1.167500 & 5.161450 & 10.16140 \\
$6,7,8,0.3,0.4$ & 1.176850 & 5.169560 & 10.16950 \\
$6,7,8,0.4,0.5$ & 1.186220 & 5.177660 & 10.17760 \\
& & & \\
$6,5,4,0.2,0.1$ & 1.333000 & 5.300180 & 10.29870 \\
$7,6,5,0.3,0.2$ & 1.231130 & 5.215260 & 10.21500 \\
$8,7,6,0.4,0.3$ & 1.174130 & 5.167170 & 10.16710 \\
$9,8,7,0.5,0.4$ & 1.139140 & 5.136310 & 10.13630 \\
& & & \\
$6,5,4,0.5,0.2$ & 1.273020 & 5.250890 & 10.25010 \\
$7,6,5,0.5,0.2$ & 1.210960 & 5.198500 & 10.19830 \\
$8,7,6,0.5,0.2$ & 1.171420 & 5.164790 & 10.16480 \\
$9,8,7,0.5,0.2$ & 1.144520 & 5.141080 & 10.14110 \\
\hline
\end{tabular}

CMRD) decrease (increase) as proportion parameters increase. On the other hand, when $\lambda_{1}>\lambda_{2}>\lambda_{3}$, hazard rates of 3-CMED, 3-CMPD, and 3-CMBD (3CMRD) increase (decrease) as component parameters increase. When $\lambda_{1}<\lambda_{2}<\lambda_{3}$, MRL of 3-CMED, 3CMPD, and 3-CMBD (3-CMRD) increase (decrease) as proportion parameters increase, whereas when $\lambda_{1}>$ $\lambda_{2}>\lambda_{3}$, the MRL of 3-CMED, 3-CMPD, and 3CMBD (3-CMRD) decrease (increase) as component parameters increase. The behavior of MWT is observed to be increasing when $\lambda_{1}<\lambda_{2}<\lambda_{3}$, and proportion parameters increase for 3-CMED, 3-CMPD, and 3CMBD. On the other hand, when $\lambda_{1}>\lambda_{2}>\lambda_{3}$, MWTs of 3-CMED, 3-CMPD, and 3-CMBD decrease as component parameters increase.

\section{References}

1. Lawless, J.F., Statistics Models and Methods for Lifetime Data, John Wiley \& Sons Inc., New Jersey (2003).

2. Lee, E.T. and Wang, J.W., Statistical Methods for Survival Data Analysis, John Wiley \& Sons Inc., New York (2003). 
3. Kleinbanm, D.G. and Klein, M., Survival Analysis, a self-learning text, Springer (2005).

4. Machin, D., Cheung, Y.B., and Parmar, M.K., Survival Analysis, a practical approach, John Wiley \& Sons Inc., New York (2006).

5. Sultan, K.S., Ismail, M.A., and Al-Moisheer, A.S. "Mixture of two inverse weibull distributions: properties and estimation", Computational Statistics \& Data Analysis, 51(1), pp. 5377-5387 (2007).

6. Kalbfleisch, J.D. and Prentice, R.L., The Statistical Analysis of Failure Time Data, John Wiley \& Sons Inc., New York (2011).

7. Chen, W.C., Hill, B.M., Greenhouse, J.B., and Fayos, J.V. "Bayesian analysis of survival curves for cancer patients following treatment", Bayesian Statistics, 2, pp. 299-328 (1985).

8. Berkson, J. and Gage, R.P. "Survival cure for cancer patients following treatment", Journal of the American Statistical Association, 47, pp. 501-515 (1952).

9. Qian, J. "A Bayesian weibull survival model", Ph.D. Thesis, Institute of Statistical and Decision Sciences, Duke University, North Carolina (1994).

10. Angelis, R.D., Capocaccia, R., Hakulinen, T., Soderman, B., and Verdecchia, A. "Mixture models for cancer survival analysis: application to populationbased data with covariates", Statistics in Medicine, 18, pp. 441-454 (1999).

11. Marin, J.M., Rodriguez-Bernal, M.T., and Wiper, M.P. "Using weibull mixture distributions to model heterogeneous survival data", Communication in Statistics-Simulation and Computation, 34, pp. 673$684(2005)$.

12. Abu-Taleb, A.A., Smadi, M.M., and Alawneh, A.J. "Bayes estimation of the lifetime parameters for the exponential distribution", Journal of Mathematics and Statistics, 3(3), pp. 106-108 (2007).

13. Erişoğlu, Ü., Erişoğlu, M., and Erol, H. "A mixture model of two different distributions approach to the analysis of heterogeneous survival data", World Academy of Science, Engineering and Technology, 54, pp. 41-45 (2011).

14. Krishna, H. and Malik, M. "Reliability estimation in Maxwell distribution with progressively type-II censored data", Journal of Statistical Computation and Simulation, 82(4), pp. 623-641 (2012).

15. Ali, S. "Mixture of the inverse Rayleigh distribution: properties and estimation in Bayesian framework", Applied Mathematical Modelling, 39(2), pp. 515-530 (2014).

16. Ateya, S.F. and Ahmad, A.E.B.A. "Inferences based on generalized order statistics under truncated type-I generalized logistic distribution", Statistics, 45(4), pp. 389-402 (2011).
17. Abushal, T.A. and Al-Zaydi, A.M. "Estimation based on generalized order statistics from a mixture of two Rayleigh distributions", International Journal of Statistics and Probability, 1(2), pp. 79-90 (2012).

18. Abushal, T.A. and Al-Zaydi, A.M. "Prediction based on generalized order statistics from a mixture of Rayleigh distributions using MCMC algorithm", Open Journal of Statistics, 2(3), pp. 356-367 (2012).

19. AL-Hussaini, E.K. and Hussein, M. "Estimation under a finite mixture of exponentiated exponential components model and balanced square error loss", Open Journal of Statistics, 2, pp. 28-38 (2012).

20. Ahmad, A.E.A. and AL-Zaydi, A.M. "Inferences under a class of finite mixture distributions based on generalized order statistics", Open Journal of Statistics, 3, pp. 231-244 (2013).

21. Ateya, S.F. "Estimation under modified Weibull distribution based on right censored generalized order statistics", Journal of Applied Statistics, 40(12), pp. 2720-2734 (2013).

22. Benaicha, H. and Chaker, A. "Weibull mixture model for reliability analysis", International Review of Electrical Engineering, 9(5), pp. 986-990 (2014).

23. El-Damcese, M.A. and Ramadan, D.A. "Analyzing system reliability using fuzzy mixture generalized linear failure rate distribution", American Journal of Mathematics and Statistics, 5(2), pp. 43-51 (2015).

24. Khan, M.N. "The modified beta Weibull distribution", Hacettepe Journal of Mathematics and Statistics, 44(6), pp. 1553-1568 (2015).

25. Ismail, A.A. "Likelihood inference for a step-stress partially accelerated life test model with type-I progressively hybrid censored data from Weibull distribution", Journal of Statistical Computation and Simulation, 84(11), pp. 2486-2494 (2014).

26. Ismail, A.A. "Reliability analysis under constant-stress partially accelerated life tests using hybrid censored data from Weibull distribution", Hacettepe Journal of Mathematics and Statistics, 45(1), pp. 181-193 (2016).

27. Acheson, M.A. and McElwee, E.M. "Concerning the reliability of electron tubes", The Sylvania Technologist, 4, pp. 38-40 (1951).

28. Tahir, M., Aslam, M., and Hussain, Z. "On the Bayesian analysis of 3-componen mixture of exponential distribution under different loss functions", Hacettepe Journal of Mathematics and Statistics, 45(2), pp. 609-628 (2016).

29. Davis, D.J. "An analysis of some failure data", Journal of the American Statistical Association, 47(258), pp. 113-150 (1952).

\section{Biographies}

Muhammad Aslam is a Professor of Statistics at Riphah International University, Islamabad, Pakistan. He has received his $\mathrm{PhD}$ degree in Statistics form 
University of Wales. He has published over 120 refereed publications. His research interests include Bayesian inference and paired comparison models.

Muhammad Tahir graduated from Quaid-i-Azam University, Islamabad, Pakistan. Currently, he is a Faculty Member at Government College University, Faisalabad, Pakistan. He has published more than 20 research papers in national and international reputed journals. His research interests include Bayesian inference, reliability analysis, and mixture distributions.

Zawar Hussain received his $\mathrm{PhD}$ degree in Statistics from the Quaid-i-Azam University, Islamabad, Pakistan. He has published 70 research papers in research journals. His research interests include sampling techniques, randomized response models, and Bayesian Statistics. 